\title{
Dynamic Observability Method for Durability Assessment Considering Measurement Noise
}

\author{
Tian Peng, Juan R. Casas and Jose Turmo \\ Department of Civil and Environmental Engineering, Universitat Politècnica de Catalunya- \\ BarcelonaTECH, Campus Nord UPC, 08034-Barcelona, Spain, \{tian.peng, joan.ramon.casas, \\ jose.turmo\}@upc.edu
}

\begin{abstract}
Due to the inevitable degradation of material properties in structures in daily use, such as stiffness degradation due to cracking in concrete elements, their durability will definitely be influenced, and their serviceability and safety could be in danger. Thus, understanding and identifying the change in the structural parameters provides new approaches to evaluate their durability. Structural system identification by dynamic observability method, which is using subsets of masses, natural frequencies and modal shapes, is a powerful tool to detect the change of structural parameters. Taking into account the presence of noise in the measurement data in real world structures, this method establishes the relative dynamic equation with the error separation items. The equation is solved by error minimization of an objective function combining the measured frequencies and mode shapes through the parameter MAC (Modal Assurance Criterion). Additionally, the algorithms and the steps are introduced based on the dynamic eigenvalue equation, which can fully demonstrate the performance of observability techniques. The present paper provides an example on how to successfully identify structural parameters. Its suitability for practical applications is demonstrated in a large frame structure. The result is a much more accurate identification of the parameters involved in the durability of the structure even in the case of noise-corrupted measurement signals
\end{abstract}

Keywords: Durability, Structural System Identification, Dynamic Observability Method, Frequencies, Mode Shapes.

\section{Introduction}

Existing structures are inevitably exposed to the natural environment (wind, earthquake, temperature difference, or even some extreme climate) and human operationt (traffic, impact, daily degradation by use). Thus, the material properties are degraded such as the bending stiffness, axial stiffness, and mass. In this case, the durability of the structures may be influenced, leading them structures into a dangerous condition instead of the originally safe state.

Regarding the durability of structures, understanding and identifying the change in the structural parameters is a great way to estimate the structure condition and safety. Structural system identification (SSI) has become a powerful tool to help engineer decision making during the life cycle of civil and infrastructures systems. SSI can be classified as the static and dynamic analysis, as well as deterministic and probabilistic methods. The former research classification depends on the type of excitation and the latter is related to the uncertainty of the parameters involved.

It is worth mentioning that the development of Observability method (OM) makes relevant contributions to deal with the remaining problem of SSI, and has been used in several engineering fields, such as hydraulics, electrical and power networks, or transportation. The 
SSI by OM under static conditions was proposed to identify 2-D beam element models in its plane, dealing with the partial observability problem and achieving an accurate assessment. While it is much easier to dynamically excite a large structure than statically, especially in large scale structures, the dynamic data are more difficult to deal with than the static one. As a result, the standard observability method used with dynamic data has shown deficient in identifying degradation processes endangering durability.

Thus, this paper proposes a new constrained dynamic observability SSI methodology, which allows the identification of a subset of characteristics of a structure, related to its durability parameters such as stiffness and mass. Subsets of natural frequencies and modal shapes are used. Firstly, the description of dynamic constrained OM method is demonstrated step by step. Secondly, the verification of this method is revealed by one example considering the measurement noise. Lastly, the potential of dynamic constrained OM when applied to large structures is shown.

\section{Methodology}

The dynamic equation of motion of a system with no damping and no external applied forces

is shown in equation (1), expressing what is the eigenvalue or characteristic value problem.

The equation can be expressed for a two-dimensional structure with $\mathrm{N}_{\mathrm{N}}$ nodes, $\mathrm{N}_{\mathrm{B}}$ boundary conditions and $\mathrm{R}$ vibration modes as:

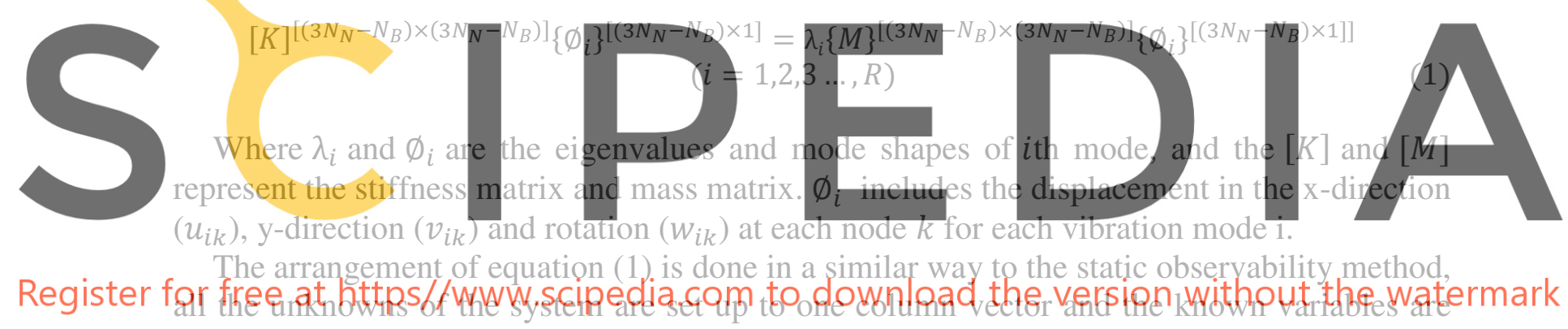

joined into a vector $\{D\}$. In this way, the previous equation might be written as:

$$
\begin{gathered}
\left.\left[B_{i}\right]\left\{z_{i}\right\}=\left[\begin{array}{ll}
K_{i, 0}^{*\left(3 N_{N}-N_{B}\right) \times r x} & \left.-M_{i, 0}^{*\left(3 N_{N}-N_{B}\right) \times m x}\right]
\end{array}\right] \begin{array}{l}
\emptyset_{K i, 0}^{* r x \times 1} \\
\emptyset_{M i, 0}^{* m x \times 1}
\end{array}\right\} \\
=\left\{M_{i, 1}^{*\left(3 N_{N}-N_{B}\right) \times n x} \emptyset_{M i, 1}^{* n x \times 1}-K_{i, 1}^{*\left(3 N_{N}-N_{B}\right) \times s x} \emptyset_{K i, 1}^{* s x \times 1}\right\}=\left\{D_{i}\right\} \\
(i=1,2,3 \ldots, R)
\end{gathered}
$$

Where $z_{i}$ represents all the unknowns, $B_{i}$ and $D_{i}$ are the corresponding coefficient matrix and augmented matrix of $i$ th mode.

Equation (2) is the description in $i$ th mode. When multiple modes are considered together, the equation will be built by combining information of several modes. For example, the first $R$ modal information is given by $[B]\{z\}=\{D\}$ shown as follows: 


$$
[B]\{z\}=\left[\begin{array}{cccc}
B_{1} & 0 & 0 & 0 \\
0 & B_{2} & 0 & 0 \\
0 & 0 & \ddots & 0 \\
0 & 0 & 0 & B_{R}
\end{array}\right]\left\{\begin{array}{c}
z_{1} \\
z_{2} \\
\vdots \\
z_{R}
\end{array}\right\}=\left[\begin{array}{cccc}
D_{1} & 0 & 0 & 0 \\
0 & D_{2} & 0 & 0 \\
0 & 0 & \ddots & 0 \\
0 & 0 & 0 & D_{R}
\end{array}\right]=\{D\}
$$

Expression in which $[B]$ is a matrix of constant coefficients, $\{D\}$ is a fully known vector and $\left\{z_{i}\right\}$ contains the full set of unknown variables. This system contains coupled and single variables. Thus, the identified coupled variables (e.g., $E I_{j} w_{i k}$ that exist in Eq.(2)) are referred as observed variables. In other to uncouple the observed variables, e.g., $E I_{j} w_{i k}=E I_{j} * w_{i k}$, the dynamic COM (constrained observability method) is here proposed based on a similar procedure as in the static case. However, in this case, the objective function is defined as:

$$
E=\sum_{i=1}^{R}\left(\Lambda f_{i}\right)^{2}+\sum_{i=1}^{R}\left(1-\frac{\left[\phi_{m i}^{T}{\overline{\phi_{m \jmath}}}^{2}\right.}{\left(\phi_{m i}^{T} \phi_{m i}\right)\left(\bar{\emptyset}_{m j} \bar{\emptyset}_{m \mathrm{j}}\right)}\right)
$$

\section{The modal assurance criterion (MAC) is used in Eq. (4), which consists of computing the} so-called MAC values as a measure for the correspondence between the calculated mode shape $\emptyset_{m i}$, obtained from the inverse analysis using the estimated stiffnesses and areas, and the measured shape $\overline{\emptyset_{m}}$ as shown in Equation (5). Besides, $\Delta f_{i}$ are the differences between the measured and estimated frequencies.
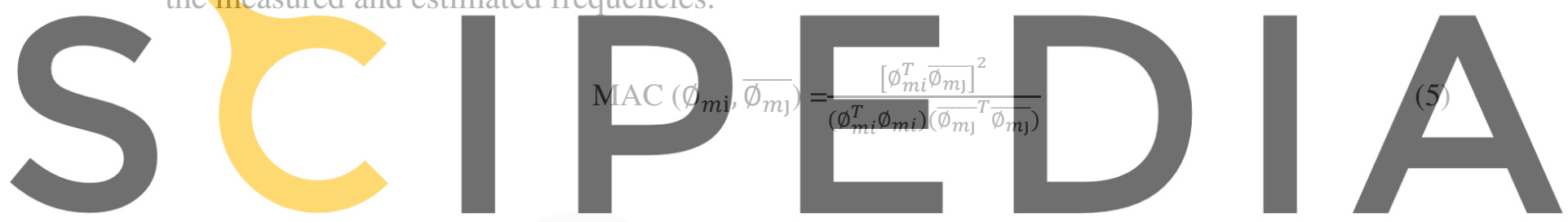

Register for free at https_AWWWAscipedia.com to download the version without the watermark

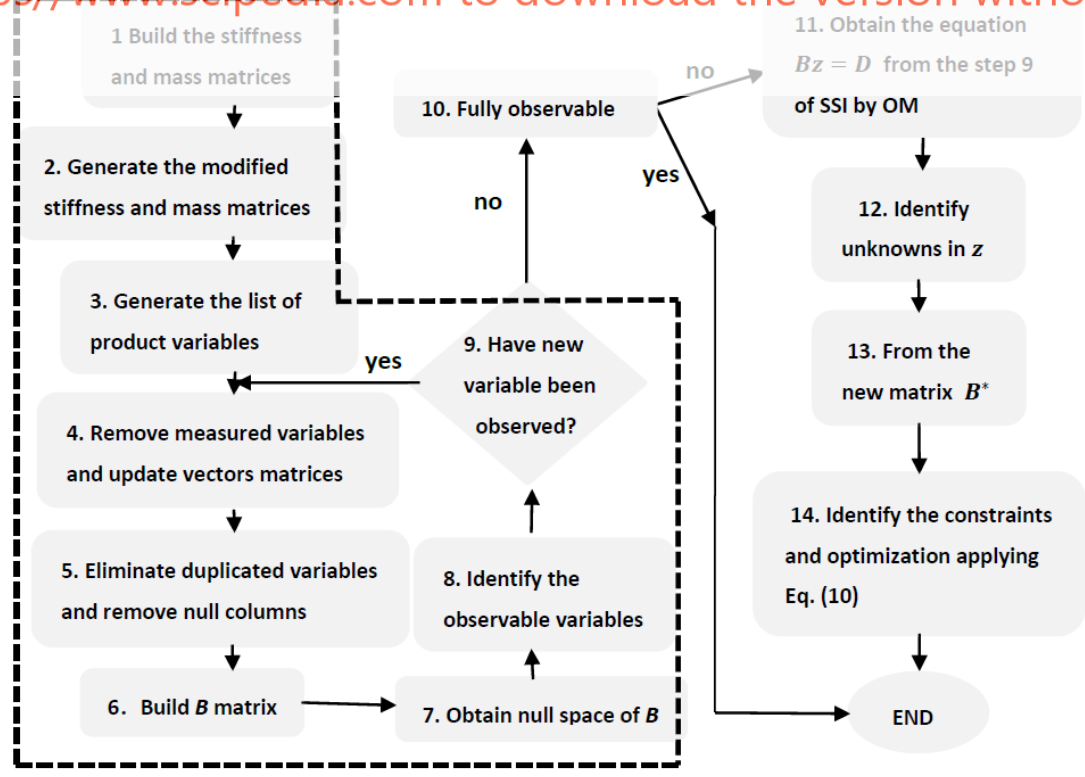

Figure 1. Flow-chart of algorithm of dynamic observability method. 
The solution is obtained by minimizing equation (4) with the imposed constraints of the form: $E A_{j} u_{i k}=E A_{j} * u_{i k}, E I_{j} v_{i k}=E I_{j} * v_{i k}, E I_{j} w_{i k}=E I_{j} * w_{i k}$ present in equation (3), which is the so-called Constrained Observability Method .

The specific steps about the method are shown in Figure.1, which is developed on the base of the static COM method.

\section{Example Analysis}

\subsection{Reinforced Concrete Beam}

The dynamic constrained observability method is verified in a reinforced concrete beam. This beam is shown in Figure 2, with a length of $6 \mathrm{~m}$ and equally divided into 30 elements, 10 parts, whose divisions can be seen minutely in Figure 2. The free vibration test of the beam was conducted by Ellen Simon, with the aim of obtaining its dynamic information, frequencies and mode shapes. The structure stiffnesses are estimated in the following way: $E I_{i}=$ $x_{i} E I_{\text {int }}, i=1 \sim 10, E I_{\text {int }}=7.23 \times 10^{6} \frac{\mathrm{N}}{\mathrm{m}^{2}}, x_{i}=[1.15,1.04,0.86,0.90,0.80,0.54,0.51,0.57$, $0.72,0.86]$ by using the experimental data after some controlled damage was produced in the beam.
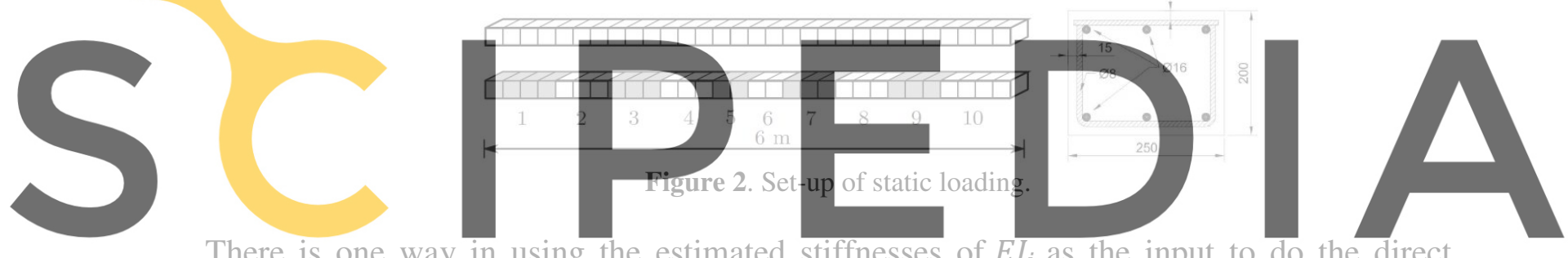

There is one way in using the estimated stiffnesses of $E I_{i}$ as the input to do the direct

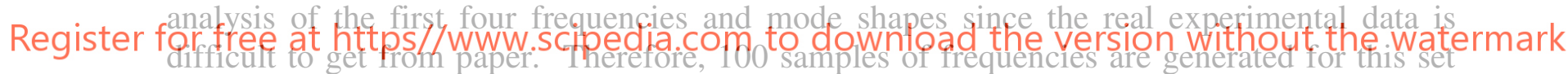
with an error of $2 \%$ while 100 samples of mode-shapes are obtained with an error of $10 \%$, because frequency accuracy obtained from dynamic tests is normally higher than for mode. One sample for a given measurement set is the collection of the associated measurements generated by Eq. (6).

$$
\tilde{\delta}=\delta\left(1+E_{\text {level }} \cdot \S\right)(\tilde{f} \text { yielded in the same way })
$$

Here, the measurements $\tilde{\delta}$ are simulated by adding proportional errors to the theoretical values, $\delta$ are obtained by direct analysis. $E_{\text {level }}$ is the error level in the measurement and $\S$ is a random number following a normal distribution with mean zero and standard deviation 0.51 to guarantee the $95 \%$ confidence interval. The samples of $\tilde{\mathrm{f}}$ are yielded in the same way. 


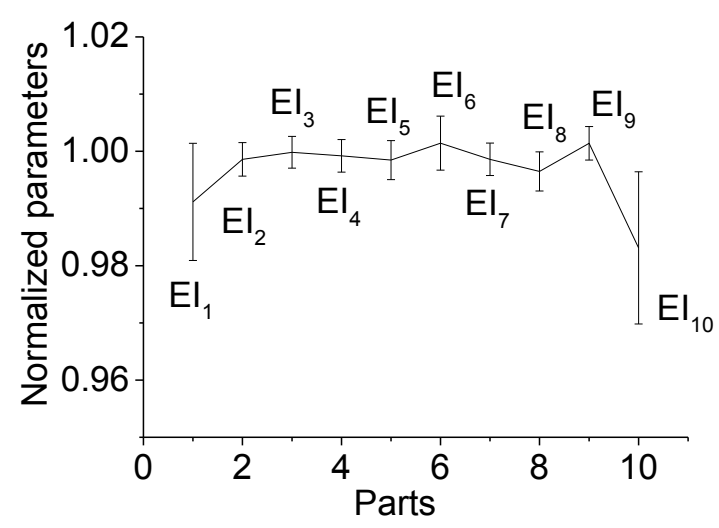

Figure 3. Mean and standard deviation of stiffness.

Figure 3 shows the estimated mean values of the 100 samples and their standard deviation for the estimated stiffness along the beam. It is shown how the errors in the mean values are less than $2 \%$ and the biggest standard deviations less than $1.5 \%$, which is a reasonable range for parameter evaluation. However, it can be obviously observed that the standard deviations of $E I_{1}$ and $E I_{10}$ are higher than other parameters, which may be due to the error accumulation from middle to the ends of the beam in the computational solution. Also the area of $E I_{1}$ and $E I_{10}$ provides little information to the solution algorithm because of their free ends. This case

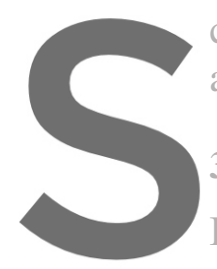
can effectively explain

actual condition of the

3.2 Example of 13-Stor

In order to show the po
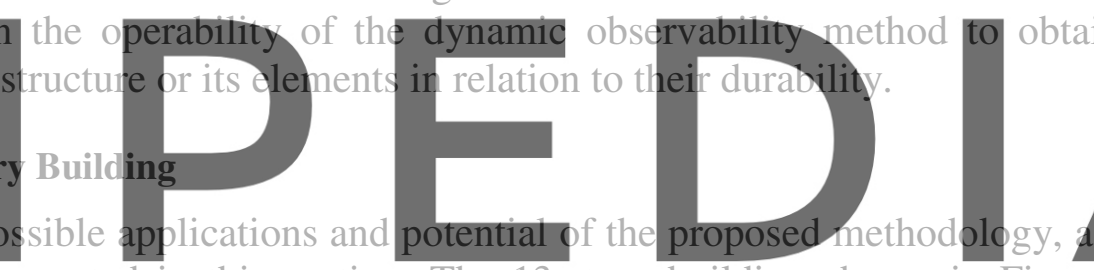

complex example is presented in this section. The 13-story building shown in Figure 4 is

Register foref under study. This structure was considered in Reference [16] this frame is modeled using a total of 226 nodes and 273 elements and it is composed or a

set of 8 different sections described in Figure 4, and the parameters are the same as in [16].

Therefore, the size of the system of equations is $678 \times 678$. The study will be performed for the first two vibration modes, which can provide relevant modal information. This structure is analyzed from a local perspective, using the constrained dynamic observability technique to only a part of the unknowns as shown in figure 4.

In fact, there are occasions in which there is interest of obtaining only the properties of one or a few members of the structure to check, for example, if it is locally damaged. This leads to a more economically efficient application of the technique. The normal procedure is to measure some of the displacements around this element or elements in order to activate its matrix components.

For this example, an assumption is made that the bending stiffnesses $E I_{1}$ and $E I_{2}$ of the structure are unknown and that the mechanical properties of the floor slab of the 12th floor are different from the theoretical one, because this part of the structure is damaged. Its modal frequency (only the first frequency is required) and mass are assumed to be known. Therefore, the properties to be observed are the bending stiffnesses of the elements between nodes 27 and 41 . 


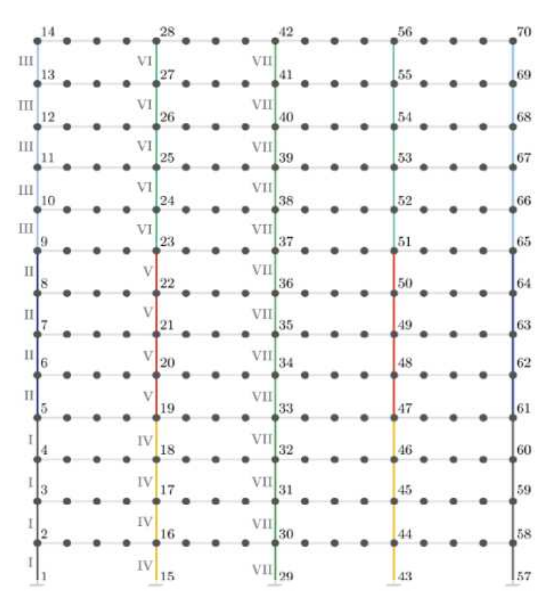

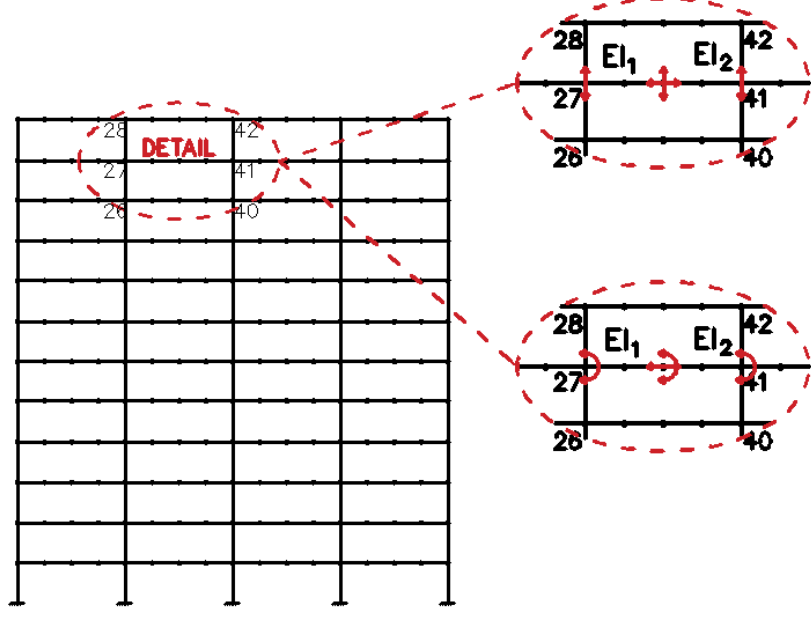

b)

b) Sets of measurements used in the local analysis.

Two sets of measurements are proposed. The first one includes the vertical displacements in three nodes and the horizontal displacement in one norle,
in Figure 4(b) (4 measurements+ Ifrequency). The secont nodes and horizontal di

measurements+ 1 frequency).

After performing the analysis.
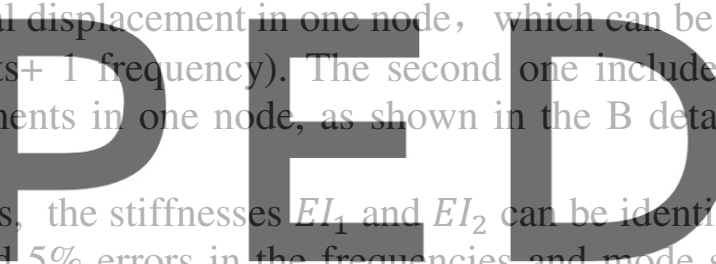

Observability assuming $2 \%$ and $5 \%$ errors in

The 100 samples are gained by Eq. (6). The estimated mean values and standard deviations of

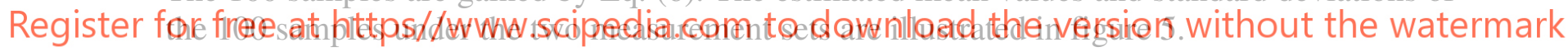

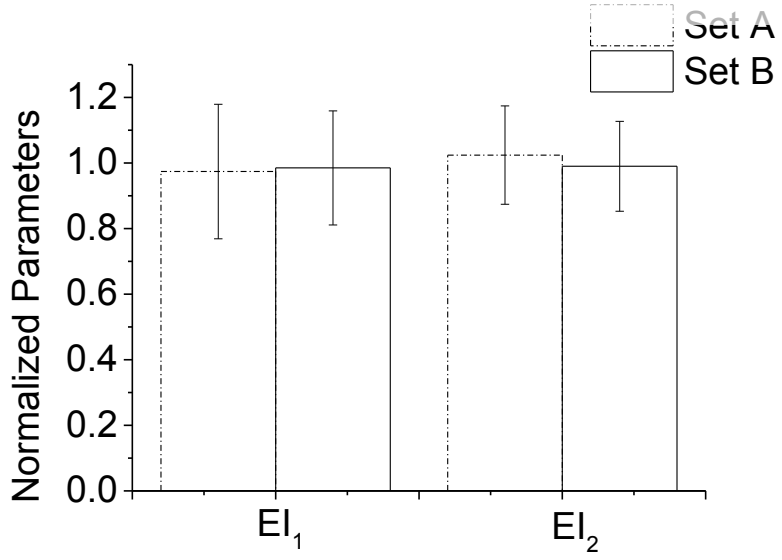

Figure 5. Mean and standard deviation of stiffness $\mathrm{EI}_{1}$ and $\mathrm{EI}_{2}$.

From figure 5, the maximum standard deviations are $2.0 \%$ and $1.7 \%$ for set $\mathrm{A}$ and set $\mathrm{B}$ 
respectively, and the mean of $E I_{1}$ and $E I_{2}$ under both sets are very close to the true value, with a $2.3 \%$ error. No matter the set of $\mathrm{A}$ or $\mathrm{B}$, the estimated value of $E I_{2}$ is a bit better than for $E I_{1}$ because of the slightly smaller standard deviations, which may be due to the stronger boundary conditions of node 41 than node 27 . It has to be emphasized that these bending stiffnesses can be observed without knowing the surrounding ones, which shows the potential of the method when applied to large structures where only a small number of measurements can be obtained. The potential application of constrained dynamic observability method to large scale structures is then fully illustrated.

\section{Conclusions}

The main objective of this paper is the application of constrained observability techniques for parametric estimation of structures using dynamic information such as frequencies and modeshapes. The final objective is to show the applicability of the method in efficiently identify the possible damages existing in the structure that may affect its durability (mainly cracking affecting the bending stiffness). A new algorithm is introduced based on the dynamic eigenvalue equation. The merit of the dynamic constrained observability analysis is demonstrated in the analysis of a RC beam and a large frame structure.

\section{Acknowledgements}

The authors are indebted to the Spanish Ministry of Economy and Competitiveness for the funding provided through the research projects BIA2013 directed by Professor José Tr that the part of this work w Mrs.Peng thorough the progr
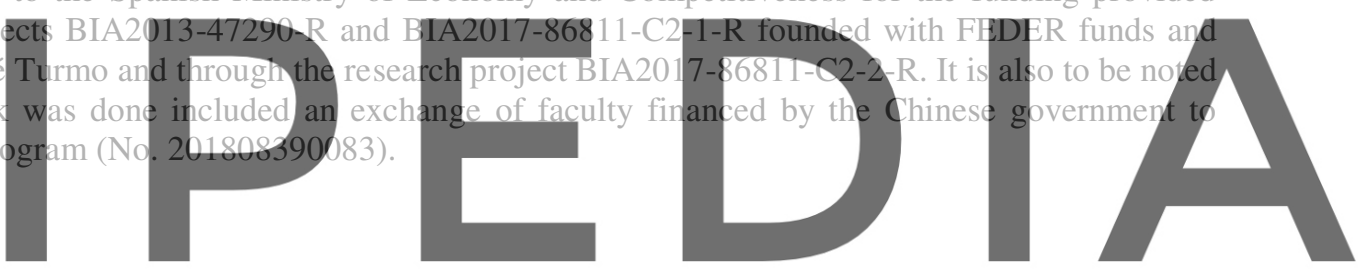

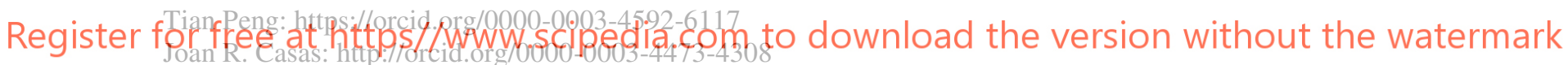
Jose Turmo: https://orcid.org/0000-0001-5001-2438

\section{References}

Bakhtiari-Nejad, F. , Rahai, A. and Esfandiari, A. (2005). A structural damage detection method using static noisy data. Engineering Structures, 27(12), 1784-1793. doi: 10.1016/j.engstruct.2005.04.019.

Castillo, E., Lozano-Galant. JA., Nogal, M. and Turmo, J. (2015). New tool to help decision making in civil engineering. Journal of Civil Engineering and Management,21:689-97. doi: 10.3846/13923730.2014.893904.

Castillo, E. , Conejo, A. J. , Pruneda, R. E. and Solares, C. (2007). Observability in linear systems of equations and inequalities: applications. Computers \& operations research,34(6), p.1708-1720. doi:10.1016/j.cor.2005.05.035.

Castillo, E. , Nogal, M. , Antonio Lozano-Galant, J. and Turmo, J. (2016). Solving some special cases of monomial ratio equations appearing frequently in physical and engineering problems. Mathematical Problems in Engineering, 2016(pt.5), 9764913.1-9764913.25. doi: 10.1155/2016/9764913.

Ellen, S., Guido, D.R. and Geert, L. (2015). Dealing with uncertainty in model updating for damage assessment: a review. Mechanical Systems \& Signal Processing, 56-57(may), 123-149. doi: 10.1016/j.ymssp.2014.11.001

Eskew, E. L. and Jang, S. (2017). Remaining stiffness estimation of buildings using incomplete measurements. Structural Control and Health Monitoring, 24:e1899. doi: 10.1002/stc.1899.

Gonzalez, Javier, Minguez, Roberto andDiaz and Sarai. (2016). Observability analysis in water transport networks: algebraic approach. Journal of Water Resources Planning \& Management. 142:4015071. 
doi:10.1061/(ASCE)WR.1943-5452.0000621.

Jin, S.S. and Jung. H.J. (2016). Sequential surrogate modeling for efficient finite element model updating. Computer \& Structures, 168:30-45. doi: 10.1016/j.compstruc.2016.02.005

Jiang, X. , Mahadevan, S. and Adeli, H. (2010). Bayesian wavelet packet denoising for structural system identification. Structural Control and Health Monitoring, 14:333-56. doi: 10.1002/stc.161.

Lei, J., Xu, D. and Turmo, J. (2018). Static structural system identification for beam-like structures using compatibility conditions. Structural Control and Health Monitoring, 25, e2062. doi: 10.1002/stc.2062.

Lei, J., Nogal, M., Lozano-Galant. JA., Xu, D. and Turmo, J. (2018). Constrained observability method in static structural system identification, Structural Control and Health Monitoring, 25.1, e2040. doi:10.1002/stc.2040.

Li Z , Park H S and Adeli H. (2016). New method for modal identification of super high-rise building structures using discretized synchrosqueezed wavelet and Hilbert transforms. The Structural Design of Tall and Special Buildings, 1-16. doi: 10.1002/tal.1312.

Lozano-Galant, JA., Nogal, M. ,Castillo, E. and Turmo, J. (2015). Selection of measurement sets in static structural identification of bridges using observability trees. Computers and Concrete, $15: 771-794$. doi: 10.12989/cac.2015.15.5.771.

Marano, G. C. Quaranta, G. and Monti, G. (2011). Modified genetic algorithm for the dynamic identification of structural systems using incomplete measurements. Computer Aided Civil \& Infrastructure Engineering, 26(2), p.92-110. doi: 10.1111/j.1467-8667.2010.00659.x.

Nogal, M., Lozano-Galant, J. A. , Turmo, J. and Castillo, E. (2016). Numerical damage identification of structures by observability techniques based on static loading tests. Structure \& Infrastructure Engineering, 12(9), 1216-1227. doi: 10.1080/15732479.2015.1101143.

Sanayei, M. and Scampoli, S. F. (1991). Structural element stiffness identification from static test data. Journal
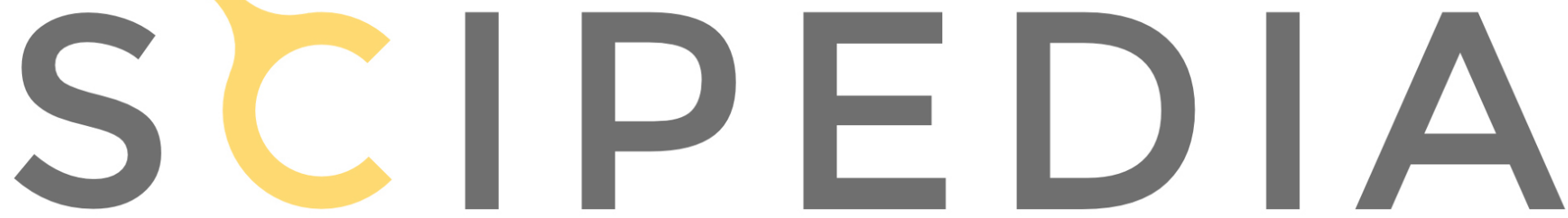

Register for free at https//www.scipedia.com to download the version without the watermark 